\title{
WAVELET CHARACTERIZATION AND MODULAR INEQUALITIES FOR WEIGHTED LEBESGUE SPACES WITH VARIABLE EXPONENT
}

\author{
Mitsuo Izuki, Eiichi Nakai and Yoshihiro Sawano \\ Okayama University, Graduate School of Education \\ Okayama 700-8530, Japan; izuki@okayama-u.ac.jp \\ Ibaraki University, Department of Mathematics \\ Mito, Ibaraki 310-8512, Japan; enakai@mx.ibaraki.ac.jp
}

Tokyo Metropolitan University, Department of Mathematics and Information Sciences Minami-Ohsawa 1-1, Hachioji-shi, Tokyo 192-0397, Japan; ysawano@tmu.ac.jp

\begin{abstract}
In this paper, we characterize weighted Lebesgue spaces with variable exponent in terms of wavelet. Also, we disprove some weighted modular inequalities when the exponent is not a constant one without using the $A_{\infty}$-condition on weights. As a byproduct, we shall obtain the vector-valued maximal inequalities in the weighted setting.
\end{abstract}

\section{Introduction}

The theory of variable exponent analysis has been rapidly developed after Kováčik and Rákosník [30] established some fundamental properties of generalized Lebesgue spaces $L^{p(\cdot)}\left(\mathbf{R}^{n}\right)$ with variable exponent $p(\cdot): \mathbf{R}^{n} \rightarrow[1, \infty]$. One of the most important problems in the theory is the boundedness of the Hardy-Littlewood maximal operator $M$ defined by

$$
M f(x):=\sup _{Q \ni x} \frac{1}{|Q|} \int_{Q}|f(y)| d y, \quad x \in \mathbf{R}^{n},
$$

for a measurable function $f: \mathbf{R}^{n} \rightarrow \mathbf{C}$, where the supremum is taken over all open cubes $Q$ containing $x$ whose sides are parallel to the coordinate axes. The boundedness of the maximal operator $M$ enabled us to analyze these function spaces more deeply. In $[6,8,10,11,14,15,16,17]$ we obtained good sufficient conditions for the boundedness of $M$ on $L^{p(\cdot)}\left(\mathbf{R}^{n}\right)$ such as the log-Hölder continuous condition (see (1.2) and (1.3) below).

For a variable exponent $p(\cdot): \mathbf{R}^{n} \rightarrow[1, \infty]$, the space $L^{p(\cdot)}\left(\mathbf{R}^{n}\right)$ is defined as the set of all measurable functions $f$ such that

$$
\|f\|_{L^{p(\cdot)}}:=\inf \{\lambda>0: \rho(f / \lambda) \leq 1\}<\infty,
$$

where

$$
\rho(f) \equiv \int_{\{p(x)<\infty\}}|f(x)|^{p(x)} d x+\|f\|_{L^{\infty}(\{p(x)=\infty\})}
$$

doi:10.5186/aasfm.2015.4032

2010 Mathematics Subject Classification: Primary 42B35, 42C40.

Key words: Muckenhoupt weight, variable exponent, wavelet, weakly positive kernel, modular inequality. 
Diening [14] and Cruz-Uribe, Fiorenza and Neugebauer [10, 11] established the following: If

$$
1<p_{-} \equiv \underset{x \in \mathbf{R}^{n}}{\operatorname{essinf}} p(x) \leq p_{+} \equiv \underset{x \in \mathbf{R}^{n}}{\operatorname{ess} \sup } p(x)<\infty,
$$

and if there exist positive constants $C$ and $p_{\infty}$ such that

$$
\begin{aligned}
& |p(x)-p(y)| \leq \frac{C}{-\log (|x-y|)}, \quad x, y \in \mathbf{R}^{n},|x-y| \leq 1 / 2, \\
& \left|p(x)-p_{\infty}\right| \leq \frac{C}{\log (e+|x|)}, \quad x \in \mathbf{R}^{n},
\end{aligned}
$$

then the Hardy-Littlewood maximal operator $M$ is bounded on $L^{p(\cdot)}\left(\mathbf{R}^{n}\right)$. Let $L H\left(\mathbf{R}^{n}\right)$ be the set of all $p(\cdot): \mathbf{R}^{n} \rightarrow[1, \infty)$ satisfying the log-Hölder continuous conditions (1.2) and (1.3).

Meanwhile the Muckenhoupt $A_{p}$ theory has been playing an important role in harmonic analysis up to now (cf. [19, 21, 38]). The $A_{p}$ condition for weight functions guarantees the boundedness of the maximal operator $M$ on weighted Lebesgue spaces $L_{w}^{p}\left(\mathbf{R}^{n}\right)$. Recently the classical Muckenhoupt $A_{p}$ class has been generalized to the setting $A_{p(\cdot)}$ of variable exponents by $[7,12,18]$ and some equivalent conditions to the boundedness of $M$ on $L_{w}^{p(\cdot)}\left(\mathbf{R}^{n}\right)$ has been given (see also [8, 27]).

For a variable exponent $p(\cdot): \mathbf{R}^{n} \rightarrow[1, \infty)$, a measurable function $w$ is said to be an $A_{p(\cdot)}$ weight if $0<w<\infty$ a.e. and

$$
\sup _{Q} \frac{1}{|Q|}\left\|w^{1 / p(\cdot)} \chi_{Q}\right\|_{L^{p(\cdot)}}\left\|w^{-1 / p(\cdot)} \chi_{Q}\right\|_{L^{p^{\prime}(\cdot)}}<\infty
$$

holds, where the supremum is taken over all open cubes $Q \subset \mathbf{R}^{n}$ whose sides are parallel to the coordinate axes and $p^{\prime}(\cdot)$ is the conjugate exponent of $p(\cdot)$, that is, $1 / p(x)+1 / p^{\prime}(x)=1$. Note that $p^{\prime}(\cdot): \mathbf{R}^{n} \rightarrow(1, \infty]$ when $p(\cdot): \mathbf{R}^{n} \rightarrow[1, \infty)$. The set $A_{p(\cdot)}$ consists of all $A_{p(\cdot)}$ weights. If $p(\cdot)$ is a constant $p$, then $A_{p(\cdot)}$ is the classical $A_{p}$ class. As is written in $[7,18]$, for $p(\cdot) \in L H\left(\mathbf{R}^{n}\right)$ satisfying $1<p_{-} \leq p_{+}<\infty$, the operator $M$ is bounded on $L_{w}^{p(\cdot)}\left(\mathbf{R}^{n}\right)$ if and only if $w \in A_{p(\cdot)}$.

In the present paper, we shall characterize weighted Lebesgue spaces $L_{w}^{p(\cdot)}\left(\mathbf{R}^{n}\right)$ in terms of wavelet assuming $p(\cdot) \in L H\left(\mathbf{R}^{n}\right)$. More precisely, we give equivalent conditions for $A_{p(\cdot)}$ by using the boundedness of an operator related to wavelets. To do this we first characterize Banach function spaces in terms of wavelet, which is achieved by the boundedness of Calderón-Zygmund operators on $L_{w}^{p(\cdot)}\left(\mathbf{R}^{n}\right)$. Finally, we give equivalent conditions for weighted modular inequalities, applying uniform boundedness of a family of operators with weakly positive kernels. We prove that $p(\cdot)$ is a constant if and only if weighted modular inequalities hold. This generalizes the result in Lerner [32] which is the case $w \equiv 1$. We do not depend upon the key observation about $A_{\infty}$-condition which is used in [32].

This article is organized as follows. In Section 2 we prepare some known results on the Hardy-Littlewood maximal operator, Banach function spaces, wavelets, weakly positive kernels, and, Muckenhoupt weights with variable exponent. In Section 3 we prove the boundedness of Calderón-Zygmund operators on $L_{w}^{p(\cdot)}\left(\mathbf{R}^{n}\right)$. To do this we prove a certain vector-valued inequality for the Hardy-Littlewood maximal operator. We will extend this inequality to a usual form in Section 5 as an appendix. 
In Section 4 we give our main results; wavelet characterization for Banach function spaces and for $L_{w}^{p(\cdot)}\left(\mathbf{R}^{n}\right)$, and, equivalent conditions for weighted modular inequalities.

In the whole paper, we will use the following notation:

1. Given a measurable set $S \subset \mathbf{R}^{n}$, we denote the Lebesgue measure by $|S|$ and the characteristic function by $\chi_{S}$.

2. Given a measurable set $S \subset \mathbf{R}^{n}$ such that $0<|S|<\infty$ and a measurable function $f$ on $\mathbf{R}^{n}$, we denote the mean value of $f$ on $S$ by $f_{S}$, namely $f_{S}:=$ $\frac{1}{|S|} \int_{S} f(x) d x$.

3. A symbol $C$ always stands for a positive constant independent of the main parameters.

4. A cube $Q \subset \mathbf{R}^{n}$ is always assumed to be open and have sides parallel to the coordinate axes. Namely we can write

$$
Q=\prod_{\nu=1}^{n}\left(x_{\nu}-r / 2, x_{\nu}+r / 2\right)
$$

using a point $x=\left(x_{1}, x_{2}, \ldots, x_{n}\right) \in \mathbf{R}^{n}$ and a constant $r>0$.

\section{Preliminaries}

2.1. Hardy-Littlewood maximal operator $M$ on $L^{p(\cdot)}\left(\mathrm{R}^{n}\right)$. In this subsection we recall some sufficient conditions for the boundedness of the HardyLittlewood maximal operator $M$ on Lebesgue spaces $L^{p(\cdot)}\left(\mathbf{R}^{n}\right)$ with variable exponent $p(\cdot): \mathbf{R}^{n} \rightarrow[1, \infty)$. Recall that the set $L H\left(\mathbf{R}^{n}\right)$ consists of all variable exponents $p(\cdot)$ satisfying the log-Hölder continuous conditions (1.2) and (1.3).

The following theorem is a sufficient condition for the boundedness of the HardyLittlewood maximal operator.

Theorem 2.1. $[5,10,11,14]$ Let $1 \leq p_{-} \leq p_{+}<\infty$. If $p(\cdot) \in \operatorname{LH}\left(\mathbf{R}^{n}\right)$, then the operator $M$ is of weak type $(p(\cdot), p(\cdot))$, that is,

$$
\left\|\chi_{\{M f>\lambda\}}\right\|_{L^{p(\cdot)}} \leq C \lambda^{-1}\|f\|_{L^{p(\cdot)}}
$$

holds for all $\lambda>0$ and all $f \in L^{p(\cdot)}\left(\mathbf{R}^{n}\right)$. Additionally if $1<p_{-}$, then $M$ is bounded on $L^{p(\cdot)}\left(\mathbf{R}^{n}\right)$, that is,

$$
\|M f\|_{L^{p(\cdot)}} \leq C\|f\|_{L^{p(\cdot)}} .
$$

For the case $p(\cdot): \mathbf{R}^{n} \rightarrow[1, \infty]$, see $[6,16]$.

We next state some equivalent conditions due to Diening [15]. Recall that $p^{\prime}(\cdot)$ means the conjugate exponent of $p(\cdot)$, that is, $1 / p(x)+1 / p^{\prime}(x)=1$ holds. Let $\mathcal{Y}$ be the set of all families of disjoint cubes in $\mathbf{R}^{n}$, and let $\mathcal{B}\left(\mathbf{R}^{n}\right)$ be the set of all variable exponent $p(\cdot)$ with $1<p_{-} \leq p_{+}<\infty$ such that the Hardy-Littlewood maximal operator $M$ is bounded on $L^{\overline{p(\cdot)}}\left(\mathbf{R}^{n}\right)$.

Theorem 2.2. [15] Let $1<p_{-} \leq p_{+}<\infty$. Then the next four conditions are equivalent:

(D1) $p(\cdot) \in \mathcal{B}\left(\mathbf{R}^{n}\right)$.

(D2) $p^{\prime}(\cdot) \in \mathcal{B}\left(\mathbf{R}^{n}\right)$.

(D3) There exists a constant $q \in\left(1, p_{-}\right)$such that $p(\cdot) / q \in \mathcal{B}\left(\mathbf{R}^{n}\right)$. 
(D4) There exists a positive constant $C>0$ such that, for all $Y \in \mathcal{Y}$ and all $f \in L^{p(\cdot)}\left(\mathbf{R}^{n}\right)$, we have

$$
\left\|\sum_{Q \in Y}|f|_{Q} \chi_{Q}\right\|_{L^{p(\cdot)}} \leq C\|f\|_{L^{p(\cdot)} .}
$$

If we take an arbitrary cube $Q$ and put $Y:=\{Q\}$ and $f:=f \chi_{Q}$ in (D4) above, then we get a weaker condition:

(A1) There exists a constant $C>0$ such that $|f|_{Q}\left\|\chi_{Q}\right\|_{L^{p(\cdot)}} \leq C\left\|f \chi_{Q}\right\|_{L^{p(\cdot)}}$ holds for all cubes $Q$ and for all $f \in L^{p(\cdot)}\left(\mathbf{R}^{n}\right)$.

In Lemma 2.4 below, we will prove the condition (A1) is a necessary condition for the weak boundedness of $M$ on $L^{p(\cdot)}\left(\mathbf{R}^{n}\right)$ and equivalent to the following condition (A2), which is called the Muckenhoupt condition for a variable exponent $p(\cdot)$ :

(A2) $\sup _{Q \text { :cube }} \frac{1}{|Q|}\left\|\chi_{Q}\right\|_{L^{p(\cdot)}}\left\|\chi_{Q}\right\|_{L^{p^{\prime}(\cdot)}}<\infty$.

See Lemmas 2.4 and 2.5 to follow, where we will prove these facts in the context of Banach function spaces.

2.2. Banach function spaces. In this subsection we first recall the definition and the fundamental properties of Banach function spaces based on the book [2]. Let $\mathcal{M}=\mathcal{M}\left(\mathbf{R}^{n}\right)$ be the set of all complex-valued measurable functions on $\mathbf{R}^{n}$.

Definition 2.1. A linear space $X \subset \mathcal{M}$ is said to be a Banach function space if there exists a functional $\|\cdot\|_{X}: \mathcal{M} \rightarrow[0, \infty]$ with the following conditions: Let $f, g, f_{j} \in \mathcal{M}(j=1,2, \ldots)$.

1. $f \in X$ if and only if $\|f\|_{X}<\infty$.

2. (Norm property):

(a) (Positivity): $\|f\|_{X} \geq 0$.

(b) (Strict positivity): $\|f\|_{X}=0$ if and only if $f=0$ a.e.

(c) (Homogeneity): $\|\lambda f\|_{X}=|\lambda| \cdot\|f\|_{X}$.

(d) (Triangle inequality): $\|f+g\|_{X} \leq\|f\|_{X}+\|g\|_{X}$.

3. (Symmetry): $\|f\|_{X}=\||f|\|_{X}$.

4. (Lattice property): If $0 \leq g \leq f$ a.e., then $\|g\|_{X} \leq\|f\|_{X}$.

5. (Fatou property): If $0 \leq f_{1} \leq f_{2} \leq \ldots$ and $\lim _{j \rightarrow \infty} f_{j}=f$ a.e., then

$$
\lim _{j \rightarrow \infty}\left\|f_{j}\right\|_{X}=\|f\|_{X}
$$

6. For all measurable sets $F$ with $|F|<\infty$, it follows $\left\|\chi_{F}\right\|_{X}<\infty$ and

$$
\int_{F}|f(x)| d x \leq C_{F}\|f\|_{X} \quad(f \in X)
$$

with the constant $C_{F}$ depending on $F$.

We need more definitions to state our main results.

Definition 2.2. A Banach function space $X$ is said to have an absolutely continuous norm, if $f, g, f_{m} \in X(m=1,2, \cdots)$ satisfy $f_{m} \rightarrow f(m \rightarrow \infty)$ a.e. $\mathbf{R}^{n}$ and $\left|f_{m}\right| \leq g$ a.e. $\mathbf{R}^{n}$ for all $m \geq 1$, then $\lim _{m \rightarrow \infty}\left\|f_{m}-f\right\|_{X}=0$. 
Definition 2.3. Let $X \subset \mathcal{M}$ be a Banach function space equipped with a norm $\|\cdot\|_{X}$. The associate space $X^{\prime}$ is defined by

$$
X^{\prime}:=\left\{f \in \mathcal{M}:\|f\|_{X^{\prime}}<\infty\right\},
$$

where

$$
\|f\|_{X^{\prime}}:=\sup \left\{\int_{\mathbf{R}^{n}}|f(x) g(x)| d x:\|g\|_{X} \leq 1\right\} .
$$

For example, the Lebesgue space $L^{p(\cdot)}\left(\mathbf{R}^{n}\right)$ with variable exponent $p(\cdot): \mathbf{R}^{n} \rightarrow$ $[1, \infty)$ is a Banach function space and the associate space is $L^{p^{\prime}(\cdot)}\left(\mathbf{R}^{n}\right)$ with norm equivalence [30].

The following lemma consists of the generalized Hölder inequality and the norm equivalence for Banach function space. See [2, pages 9-10] for example.

Lemma 2.3. Let $X \subset \mathcal{M}$ be a Banach function space.

1. For all $f \in X$ and all $g \in X^{\prime}$, we have

$$
\int_{\mathbf{R}^{n}}|f(x) g(x)| d x \leq\|f\|_{X}\|g\|_{X^{\prime}}
$$

2. For all $f \in X$ we have

$$
\sup \left\{\int_{\mathbf{R}^{n}}|f(x) g(x)| d x:\|g\|_{X^{\prime}} \leq 1\right\}=\|f\|_{X} .
$$

In particular, the space $\left(X^{\prime}\right)^{\prime}$ is equal to $X$.

As an application of the lemma above, we show the following equivalence.

Lemma 2.4. Let $X \subset \mathcal{M}$ be a Banach function space. Then the following two conditions are equivalent:

(I) $\sup _{Q: \text { cube }} \frac{1}{|Q|}\left\|\chi_{Q}\right\|_{X}\left\|\chi_{Q}\right\|_{X^{\prime}}<\infty$.

(II) There exists a positive constant $C$ such that, for all cubes $Q$ and all $f \in$ $L_{\text {loc }}^{1}\left(\mathbf{R}^{n}\right)$,

$$
|f|_{Q}\left\|\chi_{Q}\right\|_{X} \leq C\left\|f \chi_{Q}\right\|_{X}
$$

We will give a self-contained proof for readers' convenience. The same argument is found in the recent article [26].

Proof. Take an open cube $Q$ and $f \in L_{\text {loc }}^{1}\left(\mathbf{R}^{n}\right)$ arbitarily. The implication (II) $\Rightarrow(\mathrm{I})$ is proved as follows;

$$
\begin{aligned}
\frac{1}{|Q|}\left\|\chi_{Q}\right\|_{X}\left\|\chi_{Q}\right\|_{X^{\prime}} & \leq \frac{1}{|Q|}\left\|\chi_{Q}\right\|_{X} \sup \left\{\int_{\mathbf{R}^{n}}|f(x)| \chi_{Q}(x) d x:\|f\|_{X} \leq 1\right\} \\
& =\sup \left\{|f|_{Q}\left\|\chi_{Q}\right\|_{X}:\|f\|_{X} \leq 1\right\} \\
& \leq C \sup \left\{\left\|f \chi_{Q}\right\|_{X}:\|f\|_{X} \leq 1\right\} \leq C .
\end{aligned}
$$

On the other hand, from (I) and Hölder's inequality (2.1), (II) is verified;

$$
|f|_{Q}\left\|\chi_{Q}\right\|_{X}=\frac{1}{|Q|} \int_{Q}|f(y)| d y \cdot\left\|\chi_{Q}\right\|_{X} \leq \frac{1}{|Q|}\left\|f \chi_{Q}\right\|_{X}\left\|\chi_{Q}\right\|_{X^{\prime}}\left\|\chi_{Q}\right\|_{X} \leq C\left\|f \chi_{Q}\right\|_{X} .
$$

So, we are done.

The next lemma generalizes (A1). 
Lemma 2.5. If the Hardy-Littlewood maximal operator $M$ is weakly bounded on $X$, that is

$$
\left\|\chi_{\{M g>\lambda\}}\right\|_{X} \leq C \lambda^{-1}\|g\|_{X}
$$

holds for all $\lambda>0$ and all $g \in X$, then we have

$$
|f|_{Q}\left\|\chi_{Q}\right\|_{X} \leq C\left\|f \chi_{Q}\right\|_{X}
$$

for all cubes $Q$ and all $f \in L_{\text {loc }}^{1}\left(\mathbf{R}^{n}\right)$.

Proof. Take an open cube $Q$ and $f \in L_{\text {loc }}^{1}\left(\mathbf{R}^{n}\right)$ arbitrarily. If $|f|_{Q}=0$, then the conclusion is obviously true. Below we assume $|f|_{Q}>0$ and write $\lambda:=|f|_{Q} / 2$. Since $|f|_{Q} \chi_{Q}(x) \leq M\left[f \chi_{Q}\right](x)$ one has

$$
M\left[f \chi_{Q}\right]>\lambda \text { on } Q .
$$

Thus we conclude

$$
|f|_{Q}\left\|\chi_{Q}\right\|_{X} \leq|f|_{Q}\left\|\chi_{\left\{M\left[f \chi_{Q}\right]>\lambda\right\}}\right\|_{X} \leq \frac{|f|_{Q} \cdot C}{\lambda}\left\|f \chi_{Q}\right\|_{X}=C\left\|f \chi_{Q}\right\|_{X},
$$

which proves the lemma.

Below we recall some properties of weighted Banach function spaces based on Karlovich and Spitkovsky [27]. Let $X$ be a Banach function space. The set $X_{\text {loc }}$ consists of all measurable function $f$ such that $f \chi_{E} \in X$ for any measurable set $E$ with $|E|<\infty$. Given a function $W$ such that $0<W<\infty$ a.e. on $\mathbf{R}^{n}, W \in X_{\text {loc }}$ and $W^{-1} \in\left(X^{\prime}\right)_{\text {loc }}$, we define the weighted Banach function space $X\left(\mathbf{R}^{n}, W\right)$ by

$$
X\left(\mathbf{R}^{n}, W\right):=\left\{f \in \mathcal{M}\left(\mathbf{R}^{n}\right): f W \in X\right\} .
$$

Then the following is known to hold.

\section{Lemma 2.6.}

1. The weighted Banach function space $X\left(\mathbf{R}^{n}, W\right)$ is a Banach function space equipped with the norm

$$
\|f\|_{X\left(\mathbf{R}^{n}, W\right)}:=\|f W\|_{X} .
$$

2. The associate space of $X\left(\mathbf{R}^{n}, W\right)$ is $X^{\prime}\left(\mathbf{R}^{n}, W^{-1}\right)$ equipped with the norm

$$
\|f\|_{X^{\prime}\left(\mathbf{R}^{n}, W^{-1}\right)}:=\left\|f W^{-1}\right\|_{X^{\prime}} .
$$

The properties above naturally arise from those of usual Banach function spaces and the proofs are found in [27, Lemma 2.4(c)].

2.3. Wavelet and weakly positive kernels. A family of closed subspaces $\left\{V_{j}\right\}_{j \in \mathbf{Z}}$ of $L^{2}\left(\mathbf{R}^{n}\right)$ is said to be a multiresolution analysis (MRA) if the following conditions hold:

1. $V_{j} \subset V_{j+1}$ for all $j \in \mathbf{Z}$.

2. $\bigcup_{j \in \mathbf{Z}} V_{j}$ is dense in $L^{2}\left(\mathbf{R}^{n}\right)$.

3. $\bigcap_{j \in \mathbf{Z}} V_{j}=\{0\}$.

4. $f \in V_{j}$ holds if and only if $f(2 \cdot) \in V_{j+1}$ for every $j \in \mathbf{Z}$.

5. If $f \in V_{0}$, then $f(\cdot-k) \in V_{0}$ for all $k \in \mathbf{Z}^{n}$.

6. There exists a function $\varphi \in L^{2}\left(\mathbf{R}^{n}\right)$ such that $\{\varphi(\cdot-k)\}_{k \in \mathbf{Z}^{n}}$ is an orthonormal basis in $V_{0}$. 
The function $\varphi$ is said to be a scaling function of the MRA $\left\{V_{j}\right\}_{j \in \mathbf{Z}}$.

Based on the fundamental wavelet theory (cf. $[13,37,40]$ ), we can construct a compactly supported $C^{1}$ scaling function $\varphi$ of an MRA $\left\{V_{j}\right\}_{j \in \mathbf{Z}}$. For each $j \in \mathbf{Z}$ we can take a closed subspace $W_{j}$ so that $V_{j+1}=V_{j} \oplus W_{j}$. Let $L=\left\{1,2, \cdots, 2^{n}-1\right\}$. According to the standard construction, there exists a set of compactly supported $C^{1}$-functions $\left\{\psi^{l}\right\}_{l \in L}$ such that the system

$$
\left\{\psi_{j, k}^{l}:=2^{j n / 2} \psi^{l}\left(2^{j} \cdot-k\right): l \in L, k \in \mathbf{Z}^{n}\right\}
$$

is an orthonormal basis of $W_{j}$ for each $j \in \mathbf{Z}$. Thus the system

$$
\left\{\psi_{j, k}^{l}: l \in L, k \in \mathbf{Z}^{n}, j \in \mathbf{Z}\right\}
$$

becomes an orthonormal basis in $L^{2}\left(\mathbf{R}^{n}\right)$. Each function $\psi^{l}$ is called a wavelet.

Below we consider a compactly supported $C^{1}$ scaling function $\varphi$ of an MRA $\left\{V_{j}\right\}_{j \in \mathbf{Z}}$ and the associated wavelets $\left\{\psi^{l}\right\}_{l \in L}$. For each $j \in \mathbf{Z}$ the orthogonal projection $P_{j}: L^{2}\left(\mathbf{R}^{n}\right) \rightarrow V_{j}$ is given by

$$
P_{j} f(x)=\sum_{k \in \mathbf{Z}^{n}}\left\langle f, \varphi_{j, k}\right\rangle \varphi_{j, k}(x)=\int_{\mathbf{R}^{n}} K_{j}(x, y) f(y) d y,
$$

where $\varphi_{j, k}:=2^{j n / 2} \varphi\left(2^{j} \cdot-k\right)$ and

$$
\left\langle f, \varphi_{j, k}\right\rangle:=\int_{\mathbf{R}^{n}} f(x) \overline{\varphi_{j, k}(x)} d x, \quad K_{j}(x, y):=\sum_{k \in \mathbf{Z}^{n}} \varphi_{j, k}(x) \overline{\varphi_{j, k}(y)} .
$$

As we mentioned, the family $\left\{P_{j}\right\}_{j \in \mathbf{Z}}$ has some interesting properties. Because the scaling function $\varphi$ is compactly supported and bounded, we can easily prove

$$
\left|P_{j} f(x)\right| \leq C M f(x)
$$

everywhere for all $j \in \mathbf{Z}$, where $C>0$ is a constant independent of $f$ and $j$. Aimar, Bernardis and Martín-Reyes [1] has proved that (2.2) is valid for more general scaling functions. In [1] they have additionally found the next property of the family of kernels $\left\{K_{j}(x, y)\right\}_{j \in \mathbf{Z}}$. Those properties are applicable to wavelet characterizations of the usual Muckenhoupt weights and modular inequalities on Lebesgue spaces with variable exponent, see [23].

Lemma 2.7. The family $\left\{K_{j}(x, y)\right\}_{j \in \mathbf{Z}}$ enjoys the following property called weakly positive: There exist a constant $\mathcal{C}>0$ and a sequence of positive real numbers $\left\{\ell_{j}\right\}_{j \in \mathbf{Z}}$ such that

1. $0<\ell_{j+1}<\ell_{j}<\infty$ for all $j \in \mathbf{Z}$,

2. $\lim _{j \rightarrow \infty} \ell_{j}=0$ and $\lim _{j \rightarrow-\infty} \ell_{j}=\infty$,

3. $K_{j}(x, y)>\mathcal{C}\left(\ell_{j+1}\right)^{-n}$ whenever $|x-y|<\ell_{j}$.

The notion of weakly positive kernels dates back to the work by Aimar, Bernardis and Martín-Reyes [1], where they characterized the Muckenhoupt class $A_{p}$ in terms of wavelet. The notion of the weakly positve kernel is akin to the one of genuine singular integral operators. See [3, 4].

2.4. Muckenhoupt weights with variable exponent. In this subsection we define the weighted Lebesgue space $L_{w}^{p(\cdot)}\left(\mathbf{R}^{n}\right)$ with variable exponent $p(\cdot): \mathbf{R}^{n} \rightarrow$ $[1, \infty)$ and introduce a result on the boundedness of the Hardy-Littlewood maximal operator $M$ on $L_{w}^{p(\cdot)}\left(\mathbf{R}^{n}\right)$. 
Definition 2.4. For a variable exponent $p(\cdot): \mathbf{R}^{n} \rightarrow[1, \infty)$ and a function $w$ with $0<w<\infty$ a.e., the weighted Lebesgue space $L_{w}^{p(\cdot)}\left(\mathbf{R}^{n}\right)$ with variable exponent is the space $L^{p(\cdot)}\left(\mathbf{R}^{n}, w^{1 / p(\cdot)}\right)$, namely

$$
L_{w}^{p(\cdot)}\left(\mathbf{R}^{n}\right)=L^{p(\cdot)}\left(\mathbf{R}^{n}, w^{1 / p(\cdot)}\right)=\left\{f:\|f\|_{L_{w}^{p(\cdot)}}:=\left\|f w^{1 / p(\cdot)}\right\|_{L^{p(\cdot)}}<\infty\right\} .
$$

Recall that the Muckenhoupt class $A_{p(\cdot)}$ is defined by (1.4). About the properties of this class of weights, several helpful remarks may be in order.

Remark 2.1. Let $p(\cdot): \mathbf{R}^{n} \rightarrow[1, \infty)$.

1. If $p(\cdot)$ equals a constant, then the class $A_{p(\cdot)}$ is the usual Muckenhoupt class.

2. If the Hardy-Littlewood maximal operator $M$ is weak type of $(p(\cdot), p(\cdot))$, then condition (A2) is true, that is, the constant function 1 belongs to $A_{p(\cdot)}$. This fact generalizes [12, Proposition 3.8].

3. Suppose that $w_{1}, w_{2}$ are the Muckenhoupt $A_{1}$ weights and that $p(\cdot) \in L H\left(\mathbf{R}^{n}\right)$. Then $w_{1} w_{2}^{1-p(\cdot)} \in A_{p(\cdot)}$. This is the converse of Jone's factorization theorem for variable exponent, which is proved by Diening and Hästö [18].

4. By virtue of Lemmas 2.4 and 2.6, condition (1.4) is equivalent to the following:

$$
|f|_{Q}\left\|\chi_{Q}\right\|_{L_{w}^{p(\cdot)}} \leq C\left\|f \chi_{Q}\right\|_{L_{w}^{p(\cdot)}}
$$

holds for all cubes $Q$ and all $f \in L_{w}^{p(\cdot)}\left(\mathbf{R}^{n}\right)$.

The following equivalence has been proved by Diening and Hästö [18] when $p_{-}>$ 1 , where the main tools for the proof are from a series of Dieinig's work (cf. $[14,15$, 17]). We also refer to [7] for some related results. A self-contained proof including the case $p_{-}=1$ is given by Cruz-Uribe, Fiorenza and Neugebauer [12]:

Theorem 2.8. [7, 12, 18] Suppose that $p(\cdot) \in \operatorname{LH}\left(\mathbf{R}^{n}\right)$ and $p_{+}<\infty$. If $p_{-}>1$, then the following three conditions are equivalent:

(C1) $w \in A_{p(\cdot) \text {. }}$

(C2) The Hardy-Littlewood maximal operator $M$ is bounded on $L_{w}^{p(\cdot)}\left(\mathbf{R}^{n}\right)$.

(C3) $M$ is of weak type $(p(\cdot), p(\cdot))$ on $L_{w}^{p(\cdot)}\left(\mathbf{R}^{n}\right)$, namely

$$
\left\|\chi_{\{M f>\lambda\}} w^{1 / p(\cdot)}\right\|_{L^{p(\cdot)}} \leq C \lambda^{-1}\left\|f w^{1 / p(\cdot)}\right\|_{L^{p(\cdot)}}
$$

for all $\lambda>0$ and all $f \in L_{w}^{p(\cdot)}\left(\mathbf{R}^{n}\right)$.

If $p_{-} \geq 1$, then two conditions $(C 1)$ and $(C 3)$ are equivalent.

The first author has generalized Theorem 2.8 above by replacing $M$ by the family $\left\{P_{j}\right\}_{j \in \mathbf{Z}}$.

Theorem 2.9. [24] Suppose that $p(\cdot) \in L H\left(\mathbf{R}^{n}\right)$ and $p_{+}<\infty$. If $p_{-}>1$, then the following three conditions are equivalent:

(M1) $w \in A_{p(\cdot)}$.

(M2) There exists a positive constant $C$ such that

$$
\left\|\left(P_{j} f\right) w^{1 / p(\cdot)}\right\|_{L^{p(\cdot)}} \leq C\left\|f w^{1 / p(\cdot)}\right\|_{L^{p(\cdot)}}
$$

holds for all $j \in \mathbf{Z}$ and all $f \in L_{w}^{p(\cdot)}\left(\mathbf{R}^{n}\right)$.

(M3) There exists a positive constant $C$ such that

$$
\left\|\chi_{\left\{P_{j} f>\lambda\right\}} w^{1 / p(\cdot)}\right\|_{L^{p(\cdot)}} \leq C \lambda^{-1}\left\|f w^{1 / p(\cdot)}\right\|_{L^{p(\cdot)}}
$$


for all $j \in \mathbf{Z}$, all $\lambda>0$ and all $f \in L_{w}^{p(\cdot)}\left(\mathbf{R}^{n}\right)$.

If $p_{-} \geq 1$, then two conditions (M1) and (M3) are equivalent.

At the end of this subsection we state the density of $C_{\text {comp }}^{\infty}\left(\mathbf{R}^{n}\right)$ in $L_{w}^{p(\cdot)}\left(\mathbf{R}^{n}\right)$. The following is a corollary of Theorem 2.8 and [39, Theorem 1.2].

Theorem 2.10. Suppose that $p(\cdot) \in L H\left(\mathbf{R}^{n}\right)$ and $1<p_{-} \leq p_{+}<\infty$. If $w \in A_{p(\cdot)}$, then the space $C_{\text {comp }}^{\infty}\left(\mathbf{R}^{n}\right)$ is dense in $L_{w}^{p(\cdot)}\left(\mathbf{R}^{n}\right)$.

\section{Boundedness of Calderón-Zygmund operators}

Recall that an $L^{2}\left(\mathbf{R}^{n}\right)$-bounded operator $T$ is said to be a Calderón-Zygmund operator if there exists a measurable function $K$ defined on $\mathbf{R}^{n} \times \mathbf{R}^{n}$ such that for all $L^{\infty}\left(\mathbf{R}^{n}\right)$-functions with compact support we have

$$
T f(x)=\int_{\mathbf{R}^{n}} K(x, y) f(y) d y \quad \text { for all } x \notin \operatorname{supp} f
$$

and that $K$ satisfies the following estimates:

$$
|K(x, y)| \leq C \frac{1}{|x-y|^{n}},
$$

if $x \neq y$, and

$$
|K(x, z)-K(y, z)|+|K(z, x)-K(z, y)| \leq C \frac{|x-y|}{|x-z|^{n+1}},
$$

if $0<2|x-y|<|z-x|$. We aim here to prove the following result about CalderónZygmund operators.

Theorem 3.1. Suppose that $p(\cdot) \in L H\left(\mathbf{R}^{n}\right)$ and $1<p_{-} \leq p_{+}<\infty$. Let $T$ be a Calderón-Zygmund operator and $w \in A_{p(\cdot)}$. Then

$$
\|T f\|_{L_{w}^{p(\cdot)}} \leq C\|f\|_{L_{w}^{p(\cdot)}}
$$

for all $f \in L_{w}^{p(\cdot)}\left(\mathbf{R}^{n}\right)$.

For the proof, we use the Lerner decomposition. For a cube $Q$, we let $\mathcal{D}(Q)$ be the set of all dyadic cubes with respect to $Q$. For $R \in \mathcal{Q}$ and a function $f: R \rightarrow \mathbf{R}$, define

$$
\omega(f ; R):=\inf _{c \in \mathbf{R}}\left((f-c) \chi_{R}\right)^{*}\left(2^{-n-2}|R|\right),
$$

where $g^{*}$ denotes the non-increasing rearrangement of a measurable function $g$. The local dyadic sharp maximal function of $f$ is given by

$$
M_{Q}^{\sharp, d} f(x):=\sup _{R \in \mathcal{D}(Q), R \ni x} \omega(f ; R) .
$$

We invoke the following decomposition:

Lemma 3.2. [34, Theorem 4.5] Suppose that $Q_{0}$ is a cube and that $f: Q_{0} \rightarrow \mathbf{R}$ is a measurable function. Let $\left\{m_{f}(R)\right\}_{R \in \mathcal{D}\left(Q_{0}\right)} \subset \mathbf{R}$ be a collection such that

$$
\left|\left\{x \in R: f(x)>m_{f}(R)\right\}\right|,\left|\left\{x \in R: f(x)<m_{f}(R)\right\}\right| \leq \frac{1}{2}|R|
$$


for all $R \in \mathcal{D}\left(Q_{0}\right)$. Then for each $k \in \mathbf{N}$, there exists a collection $J_{k} \subset \mathcal{D}\left(Q_{0}\right)$ such that

$$
\left|\bigcup_{R \in J_{k+1}} R \cap Q\right| \leq \frac{1}{2}|Q| \quad\left(Q \in J_{k}\right), \quad \sum_{R \in J_{k+1}} \chi_{R} \leq \sum_{S \in J_{k}} \chi_{S} \leq 1
$$

for all $k=1,2, \ldots$ and that

$$
\left|f(x)-m_{f}\left(Q_{0}\right)\right| \leq 4 M_{Q_{0}}^{\sharp, d} f(x)+2 \sum_{k=1}^{\infty} \sum_{R \in J_{k}} \omega(f ; R) \chi_{R}(x)
$$

for almost all $x \in Q_{0}$.

Lemma 3.3. Let $\left(w, C_{0}\right)$ be a pair of a weight and a positive constant such that

$$
\|M f\|_{L_{w}^{p(\cdot)}} \leq C_{0}\|f\|_{L_{w}^{p(\cdot)}}
$$

for all measurable functions $f$. Then there exists $\eta>1$ such that

$$
\|M f\|_{L_{w}^{p(\cdot) / \eta}} \leq C\|f\|_{L_{w}^{p(\cdot) / \eta} .}
$$

Or equivalently, there exists $\eta>1$ such that

$$
\left\|M\left[|f|^{\eta}\right]^{1 / \eta}\right\|_{L_{w}^{p(\cdot)}} \leq C\|f\|_{L_{w}^{p(\cdot)}}
$$

Proof. Observe that by a property of $A_{1}$-weights there exists $\eta>1$ such that

$$
M\left[|G|^{\eta}\right](x) \leq C\left(C_{0}\right)[M G(x)]^{\eta}
$$

as long as $G$ is a measurable function satisfying

$$
M G(x) \leq 2 C_{0}|G(x)| \text {. }
$$

For all $f \in L_{w}^{p(\cdot) / \eta}\left(\mathbf{R}^{n}\right)$,

$$
g(x)=|f(x)|^{1 / \eta}+\sum_{j=1}^{\infty} \frac{1}{\left(2 C_{0}\right)^{j}} M^{j}\left[|f|^{1 / \eta}\right](x)
$$

is an $A_{1}$-weight, where $M^{j}$ denotes the $j$-fold composition of $M$. More precisely, the $A_{1}$ constant is less than or equal to $2 C_{0}$ :

$$
M g(x) \leq 2 C_{0} g(x) \quad\left(\text { a.e. } x \in \mathbf{R}^{n}\right) .
$$

This implies that $g$ satisfies the reverse Hölder inequality and hence

$$
M\left[g^{\eta}\right](x) \leq C M g(x)^{\eta} .
$$

Note also that $\|M f\|_{L_{w}^{p(\cdot) / \eta}}=\left(\left\|(M f)^{1 / \eta}\right\|_{L_{w}^{p(\cdot)}}\right)^{\eta}$. Consequently,

$$
\|M f\|_{L_{w}^{p(\cdot) / \eta}} \leq\left(\left\|\left(M\left[g^{\eta}\right]\right)^{1 / \eta}\right\|_{L_{w}^{p(\cdot)}}\right)^{\eta} \leq C\left(\|M g\|_{L_{w}^{p(\cdot)}}\right)^{\eta} \leq C\|f\|_{L_{w}^{p(\cdot) / \eta}}
$$

So, we are done.

Corollary 3.4. Suppose that $M$ is bounded on $L_{w}^{p(\cdot)}\left(\mathbf{R}^{n}\right)$. Then there exist $\eta>1$ and $C>0$ such that

$$
\left\|\chi_{2^{k} Q}\right\|_{L_{w}^{p(\cdot)}} \leq C 2^{k n / \eta}\left\|\chi_{Q}\right\|_{L_{w}^{p(\cdot)}}
$$

where the constant $C>0$ is independent of $Q \in \mathcal{Q}$ and $k \in \mathbf{N}$. 
Proof. Let $\eta$ be as in Lemma 3.3. Just observe

$$
2^{-k n / \eta} \chi_{2^{k} Q}(x) \leq C M \chi_{Q}(x)^{1 / \eta} \quad\left(x \in \mathbf{R}^{n}\right)
$$

for some constant $C>0$ independent of $x, Q$ and $k$.

To finish the proof of Theorem 3.1, we need the following auxiliary estimate of the vector-valued maximal inequality. Note that (3.6) is not a usual version of the Fefferman-Stein type maximal inequality. We prove the counterpart of the classical Fefferman-Stein type maximal inequality in Section 5.

Proposition 3.5. Suppose that $p \in L H\left(\mathbf{R}^{n}\right)$ and $1<p_{-} \leq p_{+}<\infty$. Suppose in addition that $r \in(1, \infty)$ and $w \in A_{p(\cdot)}$. Then

$$
\left\|\sum_{j=1}^{\infty}\left(M f_{j}\right)^{r}\right\|_{L_{w}^{p(\cdot)}} \leq C\left\|\sum_{j=1}^{\infty}\left|f_{j}\right|^{r}\right\|_{L_{w}^{p(\cdot)}}
$$

for all sequences $\left\{f_{j}\right\}_{j=1}^{\infty}$ of measurable functions.

Proof. By the duality $L_{w}^{p(\cdot)}\left(\mathbf{R}^{n}\right)-L_{w^{-p^{\prime}(\cdot) / p(\cdot)}}^{p^{\prime}(\cdot)}\left(\mathbf{R}^{n}\right)$, we can find $g \in L_{w^{-p^{\prime}(\cdot) / p(\cdot)}}^{p^{\prime}(\cdot)}\left(\mathbf{R}^{n}\right)$ with norm 1 such that

$$
\left\|\sum_{j=1}^{\infty}\left(M f_{j}\right)^{r}\right\|_{L_{w}^{p(\cdot)}} \leq C \int_{\mathbf{R}^{n}} \sum_{j=1}^{\infty} M f_{j}(x)^{r} g(x) d x .
$$

By the Fefferman-Stein type dual inequality [20], we have

$$
\int_{\mathbf{R}^{n}} \sum_{j=1}^{\infty} M f_{j}(x)^{r} g(x) d x \leq C \int_{\mathbf{R}^{n}} \sum_{j=1}^{\infty}\left|f_{j}(x)\right|^{r} M g(x) d x .
$$

By the Hölder inequality for $L_{w}^{p(\cdot)}\left(\mathbf{R}^{n}\right)$, we obtain

$$
\int_{\mathbf{R}^{n}} \sum_{j=1}^{\infty}\left|f_{j}(x)\right|^{r} M g(x) d x \leq C\left\|\sum_{j=1}^{\infty}\left|f_{j}\right|^{r}\right\|_{L_{w}^{p(\cdot)}}\|M g\|_{L_{w^{p^{\prime}(\cdot)}}^{p^{\prime}(\cdot) / p(\cdot)}} .
$$

Since $p^{\prime}(\cdot) \in L H$ and $w^{-p^{\prime}(\cdot) / p(\cdot)} \in A_{p^{\prime}(\cdot)}$, we obtain

$$
\|M g\|_{L_{w^{-p^{\prime}(\cdot) / p(\cdot)}}^{p^{\prime}(\cdot)}} \leq C\|g\|_{L_{w^{-p^{\prime}(\cdot) / p(\cdot)}}^{p^{\prime} \cdot \cdot}}=C
$$

using the boundedness of $M$ on $L_{w^{-p^{\prime}(\cdot) / p(\cdot)}}^{\left.p^{\prime} \cdot\right)}\left(\mathbf{R}^{n}\right)$. Putting (3.7)-(3.10) together, we obtain (3.6).

With these observations in mind, we prove Theorem 3.1.

Proof of Theorem 3.1. It suffices to show that $\left\|\chi_{Q_{0}} T f\right\|_{L_{w}^{p(\cdot)}} \leq C\|f\|_{L_{w}^{p(\cdot)}}$ for all cubes $Q_{0}$ and $f \in L_{\text {comp }}^{\infty}\left(\mathbf{R}^{n}\right)$, where $C$ is a constant independent of $Q_{0}$ and $f$. Observe that, whenever $x \in Q_{0}$,

$$
|T f(x)| \leq\left|m_{T f}\left(Q_{0}\right)\right|+4 M_{Q_{0}}^{\sharp, d}[T f](x)+2 \sum_{k=1}^{\infty} \sum_{Q \in J_{k}} \omega(T f ; Q) \chi_{Q}(x)
$$

by virtue of Lemma 3.2. We estimate each term. 
For the first term, we use

$$
\begin{aligned}
\left|m_{T f}\left(Q_{0}\right)\right| & =\left|m_{T f-T\left[\chi_{3 Q_{0}} f\right]+T\left[\chi_{3 Q_{0}} f\right]}\left(Q_{0}\right)\right| \\
& \leq m_{\left|T f-T\left[\chi_{3 Q_{0}} f\right]\right|+\left|T\left[\chi_{3 Q_{0}} f\right]\right|}\left(Q_{0}\right) \\
& \leq \sup _{x \in Q_{0}}\left|T f(x)-T\left[\chi_{3 Q_{0}} f\right](x)\right|+m_{\left|T\left[\chi_{3 Q_{0}} f\right]\right|}\left(Q_{0}\right) \\
& \leq C \int_{\mathbf{R}^{n} \backslash 3 Q_{0}} \frac{|f(y)| d y}{\left|c\left(Q_{0}\right)-y\right|^{n}}+m_{\left|T\left[\chi_{3 Q_{0}} f\right]\right|}\left(Q_{0}\right),
\end{aligned}
$$

where $c\left(Q_{0}\right)$ the center of $Q_{0}$. By virtue of the weak type inequality, we obtain

$$
\left|\left\{x \in Q_{0}:\left|T\left[\chi_{3 Q_{0}} f\right]\right|>\lambda\right\}\right| \leq \frac{C^{*}}{\lambda} \int_{3 Q_{0}}|f(x)| d x .
$$

Here $C^{*}$ denotes the weak- $(1,1)$ norm of $T$. If we choose

$$
\lambda=\frac{2 C^{*}}{\left|Q_{0}\right|} \int_{3 Q_{0}}|f(x)| d x
$$

then $\left|\left\{x \in Q_{0}:\left|T\left[\chi_{3 Q_{0}} f\right]\right|>\lambda\right\}\right| \leq \frac{1}{2}\left|Q_{0}\right|$ and hence

$$
m_{\left|T\left[\chi_{3 Q_{0}} f\right]\right|}\left(Q_{0}\right) \leq \lambda=\frac{2 C^{*}}{\left|Q_{0}\right|} \int_{3 Q_{0}}|f(x)| d x .
$$

By (3.11), (3.12) and the size estimate of the kernel of $T$ we have

$$
\begin{aligned}
\left|m_{T f}\left(Q_{0}\right)\right| & \leq C \int_{\mathbf{R}^{n}} \frac{|f(y)| d y}{\ell\left(Q_{0}\right)^{n}+\left|c\left(Q_{0}\right)-y\right|^{n}} \\
& \leq C \sum_{k=1}^{\infty} \frac{1}{\left|2^{k} Q_{0}\right|} \int_{2^{k} Q_{0}}|f(y)| d y \\
& \leq C \sum_{k=1}^{\infty} \frac{\left\|\chi_{2^{k} Q_{0}} w^{-1 / p(\cdot)}\right\|_{L^{p^{\prime}(\cdot)}}\|f\|_{L_{w}^{p(\cdot)}}}{\left|2^{k} Q_{0}\right|}
\end{aligned}
$$

Thus, by virtue of (1.4) and Corollary 3.4, we have

$$
\begin{aligned}
\left\|\chi_{Q_{0}}\right\|_{L_{w}^{p(\cdot)}}\left|m_{T f}\left(Q_{0}\right)\right| & \leq C \sum_{k=1}^{\infty}\left\|\chi_{Q_{0}}\right\|_{L_{w}^{p(\cdot)}} \frac{\left\|\chi_{2^{k} Q_{0}} w^{-1 / p(\cdot)}\right\|_{L^{p^{\prime}(\cdot)}}\|f\|_{L_{w}^{p(\cdot)}}}{\left|2^{k} Q_{0}\right|} \\
& \leq C \sum_{k=1}^{\infty}\left\|\chi_{Q_{0}}\right\|_{L_{w}^{p(\cdot)}} \frac{2^{k n / \eta}\left\|\chi_{Q_{0}} w^{-1 / p(\cdot)}\right\|_{L^{p^{\prime}(\cdot)}}\|f\|_{L_{w}^{p(\cdot)}}}{\left|2^{k} Q_{0}\right|} \\
& \leq C\|f\|_{L_{w}^{p(\cdot)}} .
\end{aligned}
$$

The second term can be handled by the use of the maximal operator $M$;

$$
M_{Q_{0}}^{\sharp, d}[T f](x) \leq C M f(x),
$$

see for example [33, Proposition 2.3].

It remains to deal with the last term. We set

$$
G_{k} \equiv \bigcup_{Q \in J_{k}} Q .
$$


Let $r>1$. Then we have

$$
\left\|\sum_{k=1}^{\infty} \sum_{Q \in J_{k}} \omega(T f ; Q) \chi_{Q}\right\|_{L_{w}^{p(\cdot)}} \leq 2^{r}\left\|\sum_{k=1}^{\infty} \sum_{Q \in J_{k}} \omega(T f ; Q) M\left[\chi_{Q \backslash G_{k+1}}\right]^{r}\right\|_{L_{w}^{p(\cdot)}} .
$$

By the vector-valued maximal inequality obtained in Proposition 3.5, we have

$$
\begin{aligned}
\left\|\sum_{k=1}^{\infty} \sum_{Q \in J_{k}} \omega(T f ; Q) \chi_{Q}\right\|_{L_{w}^{p(\cdot)}} & \leq 2^{r}\left\|\sum_{k=1}^{\infty} \sum_{Q \in J_{k}} \omega(T f ; Q) M\left[\chi_{Q \backslash G_{k+1}}\right]^{r}\right\|_{L_{w}^{p \cdot(\cdot)}} \\
& =2^{r}\left\|\sum_{k=1}^{\infty} \sum_{Q \in J_{k}} M\left[\omega(T f ; Q)^{1 / r} \chi_{Q \backslash G_{k+1}}\right]^{r}\right\|_{L_{w}^{p(\cdot)}} \\
& \leq C\left\|\sum_{k=1}^{\infty} \sum_{Q \in J_{k}} \omega(T f ; Q) \chi_{Q \backslash G_{k+1}}\right\|_{L_{w}^{p(\cdot)}} .
\end{aligned}
$$

Finally, by using the definition of the sharp-maximal operator, we obtain

$$
\left\|\sum_{k=1}^{\infty} \sum_{Q \in J_{k}} \omega(T f ; Q) \chi_{Q}\right\|_{L_{w}^{p(\cdot)}} \leq C\left\|M_{Q_{0}}^{\sharp, d} T f\right\|_{L_{w}^{p(\cdot)}} \leq C\|M f\|_{L_{w}^{p(\cdot)}} \leq C\|f\|_{L_{w}^{p(\cdot)}} .
$$

So we are done.

\section{Main results}

Recall $L=\left\{1,2, \cdots, 2^{n}-1\right\}$. As in Subsection 2.3 we consider a compactly supported $C^{1}$ scaling function $\varphi$ of an MRA $\left\{V_{j}\right\}_{j \in \mathbf{Z}}$, the associated wavelets $\left\{\psi^{l}\right\}_{l \in L}$ and the system

$$
\left\{\psi_{j, k}^{l}: l \in L, j \in \mathbf{Z}, k \in \mathbf{Z}^{n}\right\}
$$

which is an orthonormal basis in $L^{2}\left(\mathbf{R}^{n}\right)$. For $f \in L^{2}\left(\mathbf{R}^{n}\right)$, we define a function $V f: \mathbf{R}^{n} \rightarrow[0, \infty]$ by

$$
V f:=\left(\sum_{l \in L} \sum_{j \in \mathbf{Z}} \sum_{k \in \mathbf{Z}^{n}}\left|\left\langle f, \psi_{j, k}^{l}\right\rangle \psi_{j, k}^{l}\right|^{2}\right)^{1 / 2} .
$$

4.1. Wavelet characterization for Banach function spaces. Our main result on the boundedness property of $V$ is the following theorem:

Theorem 4.1. Let $X$ be a Banach function space on $\mathbf{R}^{n}$ such that $\psi_{j, k}^{l} \in X^{\prime}$ for every $l \in L, j \in \mathbf{Z}, k \in \mathbf{Z}^{n}$.

1. Assume the following:

(a) The space $X \cap L^{2}\left(\mathbf{R}^{n}\right)$ is dense in $X$ and the space $X^{\prime} \cap L^{2}\left(\mathbf{R}^{n}\right)$ is dense in $X^{\prime}$.

(b) The operator $V: f \mapsto V f$ is bounded both on $X$ and on $X^{\prime}$.

Then there exists a positive constant $C$ such that, for all $f \in X$,

$$
C^{-1}\|f\|_{X} \leq\|V f\|_{X} \leq C\|f\|_{X}
$$


2. If $X$ has an absolutely continuous norm and satisfies (4.1) for all $f \in X$, then the system

$$
\left\{\psi_{j, k}^{l}: l \in L, j \in \mathbf{Z}, k \in \mathbf{Z}^{n}\right\}
$$

is an unconditional basis in $X$. Additionally the wavelet expansion

$$
f=\sum_{l \in L} \sum_{j \in \mathbf{Z}} \sum_{k \in \mathbf{Z}^{n}}\left\langle f, \psi_{j, k}^{l}\right\rangle \psi_{j, k}^{l}
$$

holds for every $f \in X$, where the convergence takes place unconditionally in $X$.

Proof. Suppose both (a) and (b) hold. Then the second inequality of (4.1) follows immediately. In order to prove the first inequality, we have only to show that

$$
\left|\int_{\mathbf{R}^{n}} f(x) \overline{g(x)} d x\right| \leq C\|V f\|_{X}
$$

for all $f \in X \cap L^{2}\left(\mathbf{R}^{2}\right)$ and $g \in X^{\prime} \cap L^{2}\left(\mathbf{R}^{n}\right)$ with $\|g\|_{X^{\prime}} \leq 1$. Because $f, g \in L^{2}\left(\mathbf{R}^{n}\right)$ we get the wavelet expansions

$$
f=\sum_{l \in L} \sum_{j \in \mathbf{Z}} \sum_{k \in \mathbf{Z}^{n}}\left\langle f, \psi_{j, k}^{l}\right\rangle \psi_{j, k}^{l}, \quad g=\sum_{l \in L} \sum_{j \in \mathbf{Z}} \sum_{k \in \mathbf{Z}^{n}}\left\langle g, \psi_{j, k}^{l}\right\rangle \psi_{j, k}^{l},
$$

where the right hand sides of the above expressions converge at least in $L^{2}\left(\mathbf{R}^{n}\right)$. So, we have

$$
\left|\int_{\mathbf{R}^{n}} f(x) \overline{g(x)} d x\right|=\left|\sum_{l \in L} \sum_{j \in \mathbf{Z}} \sum_{k \in \mathbf{Z}^{n}}\left\langle f, \psi_{j, k}^{l}\right\rangle \overline{\left\langle g, \psi_{j, k}^{l}\right\rangle}\right| .
$$

By the triangle inequality and the orthonormality for the wavelet basis, we have

$$
\begin{aligned}
\left|\int_{\mathbf{R}^{n}} f(x) \overline{g(x)} d x\right| & \leq \sum_{l \in L} \sum_{j \in \mathbf{Z}} \sum_{k \in \mathbf{Z}^{n}}\left|\left\langle f, \psi_{j, k}^{l}\right\rangle \overline{\left\langle g, \psi_{j, k}^{l}\right\rangle}\right| \\
& =\sum_{l \in L} \sum_{j \in \mathbf{Z}} \sum_{k \in \mathbf{Z}^{n}}\left|\left\langle f, \psi_{j, k}^{l}\right\rangle \overline{\left\langle g, \psi_{j, k}^{l}\right\rangle}\right| \cdot\left\|\psi_{j, k}^{l}\right\|_{L^{2}}^{2} \\
& =\int_{\mathbf{R}^{n}} \sum_{l \in L} \sum_{j \in \mathbf{Z}} \sum_{k \in \mathbf{Z}^{n}}\left|\left\langle f, \psi_{j, k}^{l}\right\rangle \psi_{j, k}^{l}(x) \cdot \overline{\left\langle g, \psi_{j, k}^{l}\right\rangle \psi_{j, k}^{l}(x)}\right| d x .
\end{aligned}
$$

We abbreviate

$$
V^{l} f:=\left(\sum_{j \in \mathbf{Z}} \sum_{k \in \mathbf{Z}^{n}}\left|\left\langle f, \psi_{j, k}^{l}\right\rangle \psi_{j, k}^{l}\right|^{2}\right)^{1 / 2}, \quad V^{l} g:=\left(\sum_{j \in \mathbf{Z}} \sum_{k \in \mathbf{Z}^{n}}\left|\left\langle g, \psi_{j, k}^{l}\right\rangle \psi_{j, k}^{l}\right|^{2}\right)^{1 / 2} .
$$

Using the Schwarz inequality and the generalized Hölder inequality, we get

$$
\begin{aligned}
\left|\int_{\mathbf{R}^{n}} f(x) \overline{g(x)} d x\right| & \leq \sum_{l \in L} \int_{\mathbf{R}^{n}} V^{l} f(x) V^{l} g(x) d x \\
& \leq C\|V f\|_{X}\|V g\|_{X^{\prime}} \leq C\|V f\|_{X}\|g\|_{X^{\prime}} \leq C\|V f\|_{X} .
\end{aligned}
$$

So, the proof of (4.2) and hence (4.1) are complete.

Next we suppose that $X$ has an absolutely continuous norm and satisfies (4.1) for all $f \in X\left(\mathbf{R}^{n}\right)$. Below we write $\Lambda:=L \times \mathbf{Z} \times \mathbf{Z}^{n}$ and

$$
T_{S} f:=\sum_{(l, j, k) \in S}\left\langle f, \psi_{j, k}^{l}\right\rangle \psi_{j, k}^{l}
$$


for $S \subset \Lambda$. Based on the definition of the unconditional basis in the book [40, Definition 7.6], we have only to prove the following:

(A) There exists a positive constant $C$ such that $\left\|T_{S} f\right\|_{X} \leq C\|f\|_{X}$ for all $f \in X$ and all $S \subset \Lambda$.

(B) $\lim _{S \rightarrow \Lambda}\left\|T_{S} f-f\right\|_{X}=0$ for all $f \in X$.

Using the orthonormality of the wavelet basis and (4.1) we can easily obtain (A) as follows:

$$
\left\|T_{S} f\right\|_{X} \leq C\left\|V\left(T_{S} f\right)\right\|_{X} \leq C\|V f\|_{X} \leq C\|f\|_{X} .
$$

On the other hand, we have $V\left(f-T_{S} f\right) \leq V f \in X$ and $V\left(f-T_{S} f\right) \rightarrow 0$ as $S \rightarrow \Lambda$ by (4.1). Since $X$ is a Banach function space with the absolutely continuous norm $\|\cdot\|_{X}, \lim _{S \rightarrow \Lambda}\left\|V\left(f-T_{S} f\right)\right\|_{X}=0$ holds. Using (4.1) again we get $\left\|f-T_{S} f\right\|_{X} \leq$ $C\left\|V\left(f-T_{S} f\right)\right\|_{X}$. Namely we obtain (B).

4.2. Wavelet characterization for $L_{w}^{p(\cdot)}\left(\mathbf{R}^{n}\right)$. Based on the observations above, we prove that $A_{p(\cdot)}$ can be characterized in terms of wavelet. The theorem below has been initially proved by Lemarié-Rieusset [31] based on the boundeness of Calderón-Zygmund operator in the case of that $p(\cdot)$ is a constant.

Theorem 4.2. Suppose that $p(\cdot) \in L H\left(\mathbf{R}^{n}\right)$ and $1<p_{-} \leq p_{+}<\infty$. Then the following three conditions are equivalent:

(W1) $w \in A_{p(\cdot)}$.

(W2) There exists a positive constant $C$ such that, for all $f \in L_{w}^{p(\cdot)}\left(\mathbf{R}^{n}\right)$,

$$
C^{-1}\|f\|_{L_{w}^{p(\cdot)}} \leq\|V f\|_{L_{w}^{p(\cdot)}} \leq C\|f\|_{L_{w}^{p(\cdot)}}
$$

(W3) The system

$$
\left\{\psi_{j, k}^{l}: l \in L, j \in \mathbf{Z}, k \in \mathbf{Z}^{n}\right\}
$$

is an unconditional basis in $L_{w}^{p(\cdot)}\left(\mathbf{R}^{n}\right)$.

If this is the case, then we have the wavelet expansion

$$
f=\sum_{l \in L} \sum_{j \in \mathbf{Z}} \sum_{k \in \mathbf{Z}^{n}}\left\langle f, \psi_{j, k}^{l}\right\rangle \psi_{j, k}^{l}
$$

for every $f \in L_{w}^{p(\cdot)}\left(\mathbf{R}^{n}\right)$.

Izuki [23] and Kopaliani [29] have independently proved the theorem in the case of $w(x)=1$ applying the extrapolation theorem due to [9].

Proof of Theorem 4.2. Before beginning the proof we remark that $L_{w}^{p(\cdot)}\left(\mathbf{R}^{n}\right)$ is a Banach function space and that $\psi_{j, k}^{l} \in\left(L_{w}^{p(\cdot)}\left(\mathbf{R}^{n}\right)\right)^{\prime}$ because $\psi_{j, k}^{l}$ is continuous and compactly supported. Thus Theorem 4.1 will be applicable to $X=L_{w}^{p(\cdot)}\left(\mathbf{R}^{n}\right)$.

We first prove $(\mathrm{W} 1) \Rightarrow(\mathrm{W} 2)$. We may assume $\|f\|_{L_{w}^{p(\cdot)}}=1$. Let

$$
\Omega:=\left\{\varepsilon=\left\{\varepsilon_{j, k}^{l}\right\}_{l \in L, j \in \mathbf{Z}, k \in \mathbf{Z}^{n}}: \varepsilon_{j, k}^{l} \in\{1,-1\}\right\}
$$

and let $d \mu(\varepsilon)$ be the Bernoulli probability measure on $\Omega$. Take a finite subset $S \subset$ $L \times \mathbf{Z} \times \mathbf{Z}^{n}$ and $f \in L_{w}^{p(\cdot)}\left(\mathbf{R}^{n}\right)$ arbitrarily, and define

$$
I_{S} f:=\sum_{(l, j, k) \in S} \varepsilon_{j, k}^{l}\left\langle f, \psi_{j, k}^{l}\right\rangle \psi_{j, k}^{l} .
$$


Note that the operator $I_{S}$ is a Calderón-Zygmund operator because $\psi^{l} \in C_{\text {comp }}^{1}\left(\mathbf{R}^{n}\right)$ (see [36, 37] as well as [40, Chapter 8]). Applying Khintchine's inequality (see [28, Lemma 2] and [35]), we obtain a pointwise estimate; for $x \in \mathbf{R}^{n}$,

$$
\left(\sum_{(l, j, k) \in S}\left|\left\langle f, \psi_{j, k}^{l}\right\rangle \psi_{j, k}^{l}(x)\right|^{2}\right)^{\frac{p(x)}{2}} \leq C\left(p_{+}, p_{-}\right) \int_{\Omega}\left|I_{S} f(x)\right|^{p(x)} d \mu(\varepsilon),
$$

where $C\left(p_{+}, p_{-}\right)$is a positive constant depending only on $p_{+}$and $p_{-}$. Because $I_{S}$ is bounded on $L_{w}^{p(\cdot)}\left(\mathbf{R}^{n}\right)$ by virtue of Theorem 3.1, we have

$$
\left\|\left(\sum_{(l, j, k) \in S}\left|\left\langle f, \psi_{j, k}^{l}\right\rangle \psi_{j, k}^{l}\right|^{2}\right)^{1 / 2}\right\|_{L_{w}^{p(\cdot)}} \leq C .
$$

Thus the operator $V$ is bounded on $L_{w}^{p(\cdot)}\left(\mathbf{R}^{n}\right)$.

Meanwhile, we note that $L_{w}^{p(\cdot)}\left(\mathbf{R}^{n}\right)^{\prime}=L_{w^{-p^{\prime}(\cdot) / p(\cdot)}}^{p^{\prime}()}\left(\mathbf{R}^{n}\right)$, that $p^{\prime}(\cdot) \in L H\left(\mathbf{R}^{n}\right)$ and that $w^{-p^{\prime}(\cdot) / p(\cdot)} \in A_{p^{\prime}(\cdot)}$. Therefore using Theorem 3.1 again, we see that $V$ is bounded on $L_{w^{-p^{\prime}(\cdot) / p(\cdot)}}^{\left.p^{\prime} \cdot\right)}\left(\mathbf{R}^{n}\right)$. Moreover, from Theorem 2.10 it follows that $C_{\text {comp }}^{\infty}\left(\mathbf{R}^{n}\right)$ is dense in $L_{w}^{p(\cdot)}\left(\mathbf{R}^{n}\right)$ and in $L_{w^{-p^{\prime}(\cdot) / p(\cdot)}}^{\left.p^{\prime} \cdot\right)}\left(\mathbf{R}^{n}\right)$. Hence Theorem 4.1 gives us (W2).

Next we prove $(\mathrm{W} 2) \Rightarrow(\mathrm{W} 3)$. Note that $L_{w}^{p(\cdot)}\left(\mathbf{R}^{n}\right)$ has absolutely continuous norm. Thus if we suppose (W2), then Theorem 4.1 immediately implies (W3) and wavelet expansion (4.3).

Finally we prove (W3) $\Rightarrow(\mathrm{W} 1)$. Suppose (W3). Fix $m \in \mathbf{Z}$ and write

$$
\beta_{j, k}^{l}:= \begin{cases}1 & (j \leq m) \\ 0 & (j \geq m+1) .\end{cases}
$$

Using the truncation sequence $\left\{\beta_{j, k}^{l}\right\}_{l, j, k}$, we define

$$
Q_{m} f:=\sum_{l \in L} \sum_{j=-\infty}^{m-1} \sum_{k \in \mathbf{Z}^{n}}\left\langle f, \psi_{j, k}^{l}\right\rangle \psi_{j, k}^{l}=\sum_{l \in L} \sum_{j=-\infty}^{\infty} \sum_{k \in \mathbf{Z}^{n}} \beta_{j, k}^{l}\left\langle f, \psi_{j, k}^{l}\right\rangle \psi_{j, k}^{l}
$$

for bounded and compactly supported functions $f$. By virtue of [22, Chapter 5,

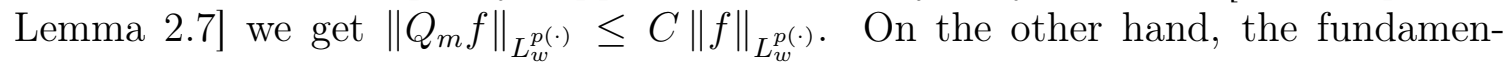
tal wavelet theory implies that $Q_{m} f=P_{m} f$ a.e. $\mathbf{R}^{n}$. Thus we have $\left\|P_{m} f\right\|_{L_{w}^{p(\cdot)}} \leq$ $C\|f\|_{L_{w}^{p(\cdot)} \text {. }}$

Now we take $g \in L_{w}^{p(\cdot)}\left(\mathbf{R}^{n}\right)$ and a cube $Q$ arbitrarily. By virtue of Lemma 2.7, there exist a constant $\mathcal{C}>0$ and a sequence of positive real numbers $\left\{\ell_{j}\right\}_{j \in \mathbf{Z}}$ such that

(1) $0<\ell_{j+1}<\ell_{j}<\infty$ for all $j \in \mathbf{Z}$,

(2) $\lim _{j \rightarrow \infty} \ell_{j}=0$ and $\lim _{j \rightarrow-\infty} \ell_{j}=\infty$,

(3) $K_{j}(x, y)>\mathcal{C}\left(\ell_{j+1}\right)^{-n}$ whenever $|x-y|<\ell_{j}$.

We can take a unique $m_{Q} \in \mathbf{Z}$ so that $\ell_{m_{Q}+1} \leq \sqrt{n}|Q|^{1 / n}<\ell_{m_{Q}}$. Then we obtain

$$
\left\|P_{m_{Q}}\left(|g| \chi_{Q}\right)\right\|_{L_{w}^{p(\cdot)}} \leq C\left\|g \chi_{Q}\right\|_{L_{w}^{p(\cdot)}} .
$$


On the other hand, we have that

$$
\begin{aligned}
\left|P_{m_{Q}}\left(|g| \chi_{Q}\right)(x)\right| \chi_{Q}(x) & =\left|\int_{Q} K_{m_{Q}}(x, y)\right| g(y)|d y| \chi_{Q}(x) \\
& \geq \int_{Q} \mathcal{C}\left(\ell_{m_{Q}+1}\right)^{-n}|g(y)| d y \cdot \chi_{Q}(x) \geq \mathcal{C} n^{-n / 2}|g|_{Q} \chi_{Q}(x) .
\end{aligned}
$$

Hence we get

$$
\left\|g \chi_{Q}\right\|_{L_{w}^{p(\cdot)}} \geq\left\|P_{m_{Q}}\left(|g| \chi_{Q}\right) \chi_{Q}\right\|_{L_{w}^{p(\cdot)}} \geq C|g|_{Q}\left\|\chi_{Q}\right\|_{L_{w}^{p(\cdot)}} .
$$

Therefore (2.3) holds, that is, (W1) is true.

\subsection{Modular inequalities.}

Theorem 4.3. Suppose that $p(\cdot) \in L H\left(\mathbf{R}^{n}\right), 1<p_{-} \leq p_{+}<\infty$ and $w \in A_{p(\cdot)}$. Then the following three conditions are equivalent:

(X1) $p(\cdot)$ is a constant.

(X2) There exists a positive constant $C$ such that, for all $j \in \mathbf{Z}$ and all $f \in$ $L_{w}^{p(\cdot)}\left(\mathbf{R}^{n}\right)$,

$$
\int_{\mathbf{R}^{n}}\left|P_{j} f(x)\right|^{p(x)} w(x) d x \leq C \int_{\mathbf{R}^{n}}|f(x)|^{p(x)} w(x) d x .
$$

(X3) There exists a positive constant $C$ such that, for all $f \in L_{w}^{p(\cdot)}\left(\mathbf{R}^{n}\right)$,

$$
C^{-1} \int_{\mathbf{R}^{n}}|f(x)|^{p(x)} w(x) d x \leq \int_{\mathbf{R}^{n}} V f(x)^{p(x)} w(x) d x \leq C \int_{\mathbf{R}^{n}}|f(x)|^{p(x)} w(x) d x .
$$

Proof. The statement $(\mathrm{X} 1) \Rightarrow(\mathrm{X} 3)$ are well known; this is just a classical result, see for example [22, Section 6.4].

Next we show that $(\mathrm{X} 2) \Rightarrow(\mathrm{X} 1)$. Suppose $(\mathrm{X} 2)$ and $p_{+}>p_{-}$. We write

$$
\begin{aligned}
\varepsilon & :=\frac{1}{3}\left(p_{+}-p_{-}\right), \\
E & :=\left\{x \in \mathbf{R}^{n}: p_{+}-\varepsilon<p(x)\right\}, \\
F & :=\left\{x \in \mathbf{R}^{n}: p_{-}+\varepsilon>p(x)\right\} .
\end{aligned}
$$

Note that $\varepsilon>0,|E|>0$ and $|F|>0$. There exist small open balls $B\left(y_{0}, r\right)=$ $\left\{x:\left|x-y_{0}\right|<r\right\}$ and $B\left(x_{0}, r\right)=\left\{x:\left|x-x_{0}\right|<r\right\}$ such that $\left|E \cap B\left(y_{0}, r\right)\right|>0$ and $\left|F \cap B\left(x_{0}, r\right)\right|>0$. We additionally define $U:=E \cap B\left(y_{0}, r\right)$ and $V:=E \cap B\left(x_{0}, r\right)$. Let $\left\{\ell_{j}\right\}$ and $\mathcal{C}$ be as in Lemma 2.7. We can take $j \in \mathbf{Z}$ so that $\ell_{j}>\left|x_{0}-y_{0}\right|+2 r$. Then we obtain

$$
P_{j}\left(\chi_{V}\right)(x)=\int_{V} K_{j}(x, y) d y \geq \mathcal{C}\left(\ell_{j+1}\right)^{-n}, \quad x \in U,
$$

where the last inequality follows by the definition of a family of weakly positive kernels and the fact that

$$
|x-y| \leq\left|x-y_{0}\right|+\left|y_{0}-x_{0}\right|+\left|x_{0}-y\right| \leq 2 r+\left|y_{0}-x_{0}\right|<\ell_{j}
$$


for every $x \in U$ and $y \in V$. Taking an arbitrary constant $R>1$, we get

$$
\begin{aligned}
R^{p_{+}-\varepsilon} \int_{U}\left(\mathcal{C}\left(\ell_{j+1}\right)^{-n}\right)^{p(x)} w(x) d x & \leq \int_{U}\left(\mathcal{C} R\left(\ell_{j+1}\right)^{-n}\right)^{p(x)} w(x) d x \\
& \leq \int_{U}\left(R P_{j}\left(\chi_{V}\right)(x)\right)^{p(x)} w(x) d x \\
& \leq \int_{\mathbf{R}^{n}}\left(R P_{j}\left(\chi_{V}\right)(x)\right)^{p(x)} w(x) d x .
\end{aligned}
$$

If we use (X2), then we have

$$
\begin{aligned}
R^{p_{+}-\varepsilon} \int_{U}\left(\mathcal{C}\left(\ell_{j+1}\right)^{-n}\right)^{p(x)} w(x) d x & \leq C \int_{\mathbf{R}^{n}}\left(R \chi_{V}(x)\right)^{p(x)} w(x) d x \\
& =C \int_{V} R^{p(x)} w(x) d x \leq C R^{p-+\varepsilon} \int_{V} w(x) d x .
\end{aligned}
$$

This contradicts $p_{+}-\varepsilon>p_{-}+\varepsilon$. Hence $p_{+}=p_{-}$holds, namely the variable exponent $p(x)$ is a constant.

Finaly, we show that $(\mathrm{X} 3) \Rightarrow(\mathrm{X} 2)$. We may assume that $f$ is bounded and compactly supported because of the density, see Theorem 2.10. Suppose (X3) and take $m \in \mathbf{Z}$ arbitrarily. We define as before

$$
Q_{m} f:=\sum_{l \in L} \sum_{j=-\infty}^{m-1} \sum_{k \in \mathbf{Z}^{n}}\left\langle f, \psi_{j, k}^{l}\right\rangle \psi_{j, k}^{l} .
$$

Then $P_{m} f(x)=Q_{m} f(x)$ for almost every $x \in \mathbf{R}^{n}$. Thus we get

$$
\begin{aligned}
\int_{\mathbf{R}^{n}}\left|P_{m} f(x)\right|^{p(x)} w(x) d x & =\int_{\mathbf{R}^{n}}\left|Q_{m} f(x)\right|^{p(x)} w(x) d x \\
& \leq C \int_{\mathbf{R}^{n}}\left|V\left(Q_{m} f\right)(x)\right|^{p(x)} w(x) d x \\
& \leq C \int_{\mathbf{R}^{\mathbf{n}}}\left(\sum_{l \in L} \sum_{j=-\infty}^{m-1} \sum_{k \in \mathbf{Z}^{n}}\left|\left\langle f, \psi_{j, k}^{l}\right\rangle \psi_{j, k}^{l}(x)\right|^{2}\right)^{\frac{p(x)}{2}} w(x) d x \\
& \leq C \int_{\mathbf{R}^{n}} V f(x)^{p(x)} w(x) d x \leq C \int_{\mathbf{R}^{n}}|f(x)|^{p(x)} w(x) d x .
\end{aligned}
$$

This implies that (X2) holds.

Remark 4.1. Lerner [32] has proved that if $p(\cdot)$ satisfies $1<p_{-} \leq p_{+}<\infty$ and

$$
\int_{\mathbf{R}^{n}} M f(x)^{p(x)} d x \leq C \int_{\mathbf{R}^{n}}|f(x)|^{p(x)} d x
$$

for all $f$, then $p(\cdot)$ equals a constant. Lerner's proof depends on the Muckenhoupt $A_{\infty}$ property. By a similar argument the first author [23] has proved Theorem 4.3 in the case of $w \equiv 1$. Our proof of Theorem 4.3 above is based on straightforward calculation and does not need the Muckenhoupt $A_{\infty}$ property. 


\section{Appendix. Fefferman-Stein vector-valued inequality for weighted Lebesgue spaces with variable exponent}

We can prove the following vector-valued inequality:

Proposition 5.1. Suppose that $p(\cdot) \in L H\left(\mathbf{R}^{n}\right)$ and $1<p_{-} \leq p_{+}<\infty$. Suppose in addition that $r \in(1, \infty]$ and $w \in A_{p(\cdot)}$. Then

$$
\left\|\left(\sum_{j=1}^{\infty}\left(M f_{j}\right)^{r}\right)^{\frac{1}{r}}\right\|_{L_{w}^{p(\cdot)}} \leq C\left\|\left(\sum_{j=1}^{\infty}\left|f_{j}\right|^{r}\right)^{\frac{1}{r}}\right\|_{L_{w}^{p(\cdot)}}
$$

for all sequences $\left\{f_{j}\right\}_{j=1}^{\infty}$ of measurable functions. When $r=\infty$, (3.5) reads

$$
\left\|\sup _{j \in \mathbf{N}} M f_{j}\right\|\left\|_{L_{w}^{p(\cdot)}} \leq C\right\| \sup _{j \in \mathbf{N}}\left|f_{j}\right| \|_{L_{w}^{p(\cdot)}} .
$$

Proof. If $r=\infty$, then we can resort to Theorem 2.8 and a trivial inequality

$$
\sup _{j \in \mathbf{N}} M f_{j} \leq M\left[\sup _{j \in \mathbf{N}}\left|f_{j}\right|\right] \text {. }
$$

Next, by Lemma 3.3 there exists $\eta>1$ such that $w \in A_{p(\cdot) / \eta}$. Let $1<r<\min \left(\eta, p_{-}\right)$. Then $p(\cdot) / r \in L H, 1<p_{-} / r \leq p_{+} / r<\infty$ and $w \in A_{p(\cdot) / \eta} \subset A_{p(\cdot) / r}$. By the definition of the weighted norm and Proposition 3.5, we have

$$
\begin{aligned}
\left\|\left(\sum_{j=1}^{\infty}\left(M f_{j}\right)^{r}\right)^{\frac{1}{r}}\right\|_{L_{w}^{p(\cdot)}} & =\left(\left\|\sum_{j=1}^{\infty}\left(M f_{j}\right)^{r}\right\|_{L_{w}^{p(\cdot) / r}}\right)^{\frac{1}{r}} \\
& \leq C\left(\left\|\sum_{j=1}^{\infty}\left|f_{j}\right|^{r}\right\|_{L_{w}^{p(\cdot) / r}}\right)^{\frac{1}{r}}=C\left\|\left(\sum_{j=1}^{\infty}\left|f_{j}\right|^{r}\right)^{\frac{1}{r}}\right\|_{L_{w}^{p(\cdot)}} .
\end{aligned}
$$

So we are done in the case where $r$ is small enough.

We let $L_{w}^{p(\cdot)}\left(\ell^{r}, \mathbf{R}^{n}\right)$ be the set of all sequences $\left\{f_{j}\right\}_{j=1}^{\infty}$ of measurable functions such that

$$
\left\|\left\{f_{j}\right\}_{j=1}^{\infty}\right\|_{L_{w}^{p(\cdot)}\left(\ell^{r}\right)}\left\|\left(\sum_{j=1}^{\infty}\left|f_{j}\right|^{r}\right)^{\frac{1}{r}}\right\|_{L_{w}^{p(\cdot)}}
$$

is finite. Note that $\left(L_{w}^{p(\cdot)}\left(\ell^{r}, \mathbf{R}^{n}\right),\|\cdot\|_{L_{w}^{p(\cdot)}\left(\ell^{r}\right)}\right)$ is a Banach space.

For the remaining case, we consider a linear operator

$$
U:\left\{f_{j}\right\}_{j=1}^{\infty} \mapsto\left\{\frac{1}{r_{j}(\cdot)^{n}} \int_{B\left(\cdot, r_{j}(\cdot)\right)} f_{j}(y) d y\right\}_{j=1}^{\infty},
$$

where each $r_{j}(\cdot)$ is a positive measurable function. We know that $U$ is bounded from $L_{w}^{p(\cdot)}\left(\ell^{r}, \mathbf{R}^{n}\right)$ to itself when $r=\infty$ or $r$ is sufficiently close to 1 . Thus, we are in the position of using the interpolation theorem [21, p. 492, Theorem 3.4] to conclude that the vector-valued inequality is valid for all $1<r \leq \infty$.

Acknowledgment. Mitsuo Izuki was partially supported by Grant-in-Aid for Scientific Research (C), No. 24540185, Japan Society for the Promotion of Science. 
Eiichi Nakai was partially supported by Grant-in-Aid for Scientific Research (C), No. 24540159, Japan Society for the Promotion of Science. Yoshihiro Sawano was partially supported by Grant-in-Aid for Young Scientists (B), No. 24740085, Japan Society for the Promotion of Science.

\section{References}

[1] Aimar, H. A., A. L. Bernardis, and F. J. Martín-Reyes: Multiresolution approximations and wavelet bases of weighted $L^{p}$ spaces. - J. Fourier Anal. Appl. 9, 2003, 497-510.

[2] Bennett, C., and R. Sharpley: Interpolation of operators. - Academic Press, Boston, San Diego, New York, 1988.

[3] Burenkov, V. I., V.S. Guliyev, A. Serbetci, and T. V. Tararykova: Necessary and sufficient conditions for the boundedness of genuine singular integral operators in local Morreytype spaces. - Dokl. Math. 78:2, 2008, 651-654; Transl. from Dokl. Ross. Akad. Nauk 422:1, 2008, 11-14.

[4] Burenkov, V.I., V.S. Guliyev, A. Serbetci, and T. V. Tararykova: Necessary and sufficient conditions for the boundedness of genuine singular integral operators in local Morreytype spaces. - Eurasian Math. J. 1:1, 2010, 32-53.

[5] Capone, C., D. Cruz-Uribe, and A. Fiorenza: The fractional maximal operator and fractional integrals on variable $L^{p}$ spaces. - Rev. Mat. Iberoam. 23:3, 2007, 743-770.

[6] Cruz-Uribe, D., L. Diening, and A. Fiorenza: A new proof of the boundedness of maximal operators on variable Lebesgue spaces. - Bull. Unione Mat. Ital. (9) 2:1, 2009, 151-173.

[7] Cruz-Uribe, D., L. Diening, and P. HÄstö: The maximal operator on weighted variable Lebesgue spaces. - Fract. Calc. Appl. Anal. 14, 2011, 361-374.

[8] Cruz-Uribe, D. V., and A. Fiorenza: Variable Lebesgue spaces: Foundations and harmonic analysis. - Birkhäuser, 2013.

[9] Cruz-Uribe, D., A. Fiorenza, J. M. Martell, and C. PÉrez: The boundedness of classical operators on variable $L^{p}$ spaces. - Ann. Acad. Sci. Fenn. Math. 31, 2006, 239-264.

[10] Cruz-Uribe, D., A. Fiorenza, and C. J. Neugebauer: The maximal function on variable $L^{p}$ spaces. - Ann. Acad. Sci. Fenn. Math. 28, 2003, 223-238.

[11] Cruz-Uribe, D., A. Fiorenza, and C. J. Neugebauer: Corrections to "The maximal function on variable $L^{p}$ spaces". - Ann. Acad. Sci. Fenn. Math. 29, 2004, 247-249.

[12] Cruz-Uribe, D., SFO, A. Fiorenza, and C. J. Neugebauer: Weighted norm inequalities for the maximal operator on variable Lebesgue spaces. - J. Math. Anal. Appl. 394, 2012, 744760 .

[13] Daubechies, I.: Orthonormal basis of compactly supported wavelets. - Comm. Pure Appl. Math. 41, 1988, 909-996.

[14] Diening, L.: Maximal function on generalized Lebesgue spaces $L^{p(\cdot)}$. - Math. Inequal. Appl. 7, 2004, 245-253.

[15] Diening, L.: Maximal functions on Musielak-Orlicz spaces and generalized Lebesgue spaces. - Bull. Sci. Math. 129, 2005, 657-700.

[16] Diening, L., P. Harjulehto, P. Hästö, Y. Mizuta, and T. Shimomura: Maximal functions in variable exponent spaces: limiting cases of the exponent. - Ann. Acad. Sci. Fenn. Math. $34,2009,503-522$.

[17] Diening, L., P. Harjulehto, P. HÄstö, and M. RưŽIČKA: Lebesgue and Sobolev spaces with variable exponents. - Lecture Notes in Math. 2017, Springer, Heidelberg, 2011.

[18] Diening, L., and P. HÄstö: Muckenhoupt weights in variable exponent spaces. - Preprint, 2010. 
[19] Duonndikoetxea, J.: Fourier analysis. - Amer. Math. Soc., Providence, RI, 2001.

[20] Fefferman, C., and E. M. Stein: Some maximal inequalities. - Amer. J. Math. 93, 1971, $107-115$.

[21] García-Cuerva, J., and J. L. Rubio de Francia: Weighted norm inequalities and related topics. - North-Holland Publishing Co., Amsterdam, 1985.

[22] Hernández, E. and G. Weiss: A first course on wavelets. - Stud. Adv. Math., CRC Press, Boca Raton, FL, 1996.

[23] Izuki, M.: Wavelets and modular inequalities in variable $L^{p}$ spaces. - Georgian Math. J. 15, 2008, 281-293.

[24] Izuki, M.: Remarks on Muckenhoupt weights with variable exponent. - J. Anal. Appl. 11, $2013,27-42$.

[25] Izuki, M., E. NakaI, and Y. Sawano: Function spaces with variable exponents - an introduction. - Sci. Math. Jpn. 77:2, 2014, 187-315.

[26] Izuki, M., Y. Sawano, and Y. Tsutsui: Variable Lebesgue norm estimates for BMO functions II. - Anal. Math. 40, 2014, 215-230.

[27] Karlovich, A. Yu., and I. M. Spitkovsky: The Cauchy singular integral operator on weighted variable Lebesgue spaces. - In: Concrete operators, spectral theory, operators in harmonic analysis and approximation, Oper. Theory Adv. Appl. 236, Birkhäuser/Springer, Basel, 2014, 275-291.

[28] Khintchine, A.: Über dyadische Brüche. - Math. Z. 18:1, 1923, 109-116.

[29] Kopaliani, T. S.: Greediness of the wavelet system in $L^{p(t)}(\mathbf{R})$. - East J. Approx. 14, 2008, $59-67$.

[30] Kováčik, O., and J. Rákosník: On spaces $L^{p(x)}$ and $W^{k, p(x)}$ - Czechoslovak Math. 41:116, $1991,592-618$.

[31] Lemarié-Rieusset, P.: Ondelettes et poids de Muckenhoupt. - Studia Math. 108, 1994, $127-147$.

[32] Lerner, A. K.: On modular inequalities in variable $L^{p}$ spaces. - Arch. Math. 85, 2005, 538543.

[33] Lerner, A. K.: A simple proof of the $A_{2}$ conjecture. - Int. Math. Res. Not. 14, 2013, 31593170.

[34] Lerner, A. K.: On an estimate of Calderón-Zygmund operators by dyadic positive operators. - J. Anal. Math. 121, 2013, 141-161.

[35] Lindenstrauss, J., and L. Tzafriri: Classical Banach spaces I and II. - Springer-Verlag, Berlin, Hedelberg, 1996.

[36] Meyer, Y.: Ondelettes et operateurs I. - Hermann, Paris, 1990.

[37] Meyer, Y.: Wavelets and operators. - Cambridge Univ. Press, Cambridge, 1992.

[38] Muckenhoupt, B.: Weighted norm inequalities for the Hardy maximal function. - Trans. Amer. Math. Soc. 165, 1972, 207-226.

[39] Nakai, E., N. Tomita, and K. Yabuta: Density of the set of all infinitely differentiable functions with compact support in weighted Sobolev spaces. - Sci. Math. Jpn. 60, 2004, 121127.

[40] Wojtaszczyk, P.: A mathematical introduction to wavelets. - Cambridge Univ. Press, Cambridge, 1997. 